\title{
LA FEMELLE DE KHALILIA RHINOCEROTIS NEVEU-LEMAIRE PARASITE DU RHINOCÉROS AFRICAIN (RHINOCEROS BICORNIS)
}

\author{
Par M. NEVEU-LEMAIRE
}

Dans une récente étude sur les Strongylidés du rhinocéros africain, Rhinoceros bicornis (1), nous avons cru devoir réunir dans une sous-famille particulière, à laquelle nous avons donné le nom d'Amirinx, un certain nombre de formes constituant, par la conformation de la bourse caudale et la disposition de l'apparei! génital femelle, un groupe intermédiaire entre les Bunostominæ et les Cylicostominæ, tels que les comprennent Railliet et Henry.

Dans cette sous-famille se placent deux genres : $1^{\circ}$ le genre Amira Lane, 1914, dont l'espèce type est A. pileata (Railliet, Henry et Bauche, 1914) de l'éléphant d'Asie ; $2^{\circ}$ le genre Khalilia NeveuLemaire, 1924, ayant pour type $K$. rhinocerotis Neveu-Lemaire, 1924, du rhinocéros africain et renfermant une autre espèce, K. sameera (Khalil, 1922), syn. : Amira semeera Khalil, 1922, de l'éléphant d'Afrique.

Or, dans la description de Khalilia rhinocerotis, nous avons été très bref en ce qui concerne la femelle, que nous n'avons découverte qu'au cours de l'impression de notre article, ce qui ne nous a pas permis de la figurer ; c'est pourquoi nous revenons ici sur cette intéressante espèce, désirant en donner des figures, accompagnées de quelques détails complémentaires.

La femelle de $K$. rhinocerotis est d'ailleurs la seule femelle du genre Khalilia connue jusqu'ici, car Khalil n'a vu que le mâle de $K$. sameera. Par contre, on connaît la femelle d'Amira pileata décrite par Railliet, Henry et Bauche en 1914.

(1) Neveu-Lemaine (M.). - Les Strongylidés du rhinocéros africain (Rhinoceros bicornis). Annales de Parasitologie humaine et comparée, II. No 2, avril 1924, p. 121-153. Dans ce-mémoire, nous avons omis de citer, parmi les parasites des rhinocéros, trois nématodes signalés récemment par Baylis et Daubney (Records of the Indian Museum, XXV, Part. VI, p. 563) et rencontrés dans l'estomac du rhinocéros indien, Rhinoceros indicus, à Nepal terai, Inde. Ce sont :

Crossocephalus brevicaudatus Baylis et Daubney, 1923 (trois femelles).

Kiluluma stylosa (von Linstow, 1907), déjà rencontré dans l'estomac du rhinocéros africain (nombreux spécimens).

Necator americanus (W. Stiles, 1902) (une femelle). Ce dernier paraît être un parasite erratique.

AnNales de Parasitologie, T, II, No 3 , - Juillet 1924, p. 224. 
Tome II. Pl. XXI.

MÉmoIre NeveU-Lemaire
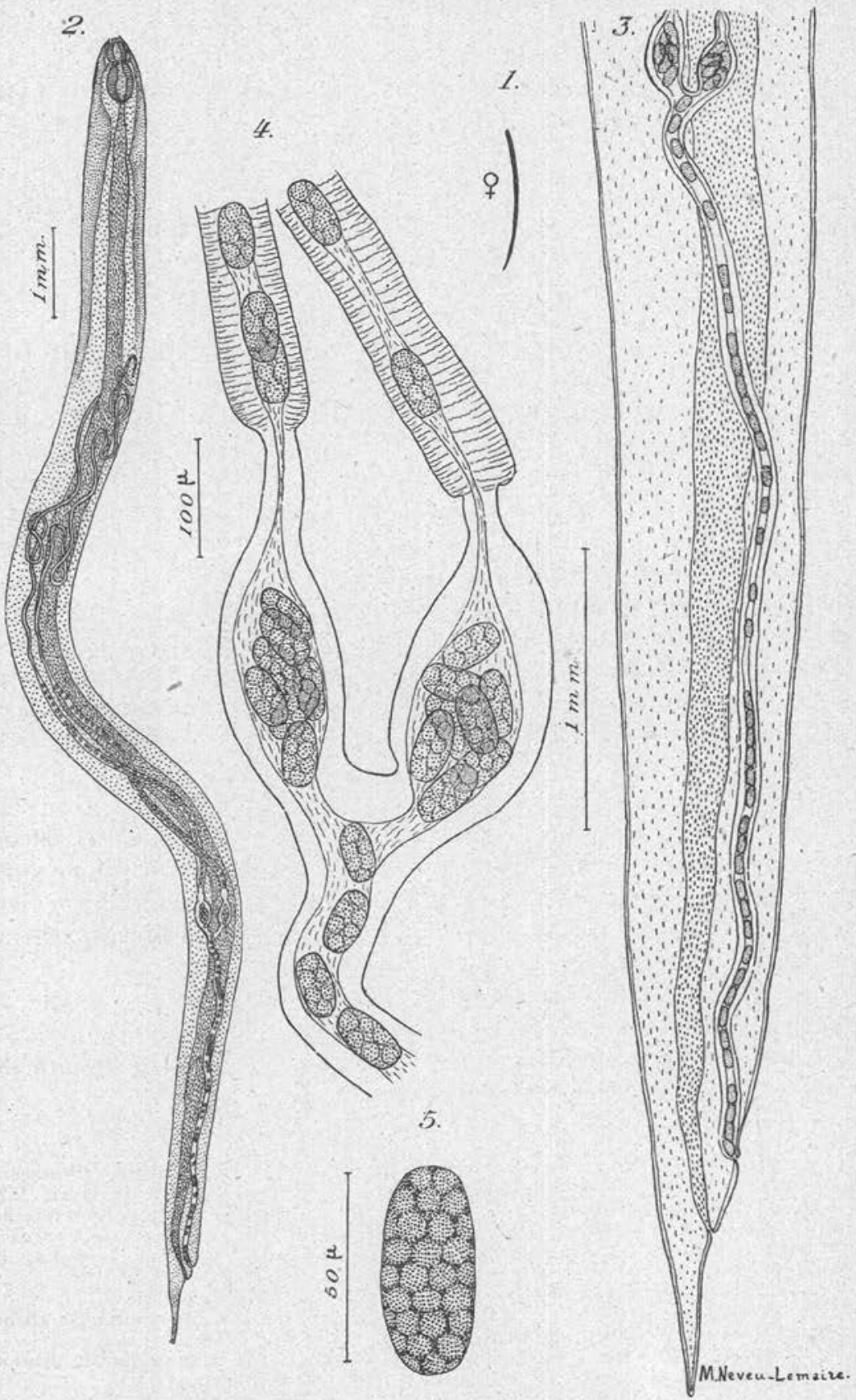

Khalidia rhinocerotis Neveu-Lemaire, 1924 ; femelle, 

La femelle de $K$. rhinocerotis, obtuse à l'extrémité antérieure et légèrement atténuée à l'extrémité postérieure, est sensiblement plus longue que le mâle et mesure 18 millimètres sur une largeur maxima de $950 \mu$. Elle se termine postérieurement par une petite pointe en avant de laquelle débouche l'anus, situé à $600 \mu$ de l'extrémité caudale. La vulve est très rapprochée de l'anus et n'en est séparée que par une distance de $260 \mu$. La disposition de l'appareil génital mérite d'attirer l'attention (PI. XXI). Les ovaires décrivent quelques sinuosités, dépassant le tiers antérieur du corps, puis descendent parallèlement et se continuent par deux utérus également parallèles. Vers le quart postérieur du corps, les utérus aboutissent à deux ovéjecteurs bien développés, d'où part, après leur réunion, un très long vagin mesurant $3 \mathrm{~mm}$., 6 . Les utérus, les ovéjecteurs et le vagin contiennent de nombreux œufs ovoïdes allongés, longs de $50 \mu$ sur $30 \mu$ de large et en voie de segmentation.

Cette disposition de l'appareil génital femelle, en particulier la grande longueur du vagin, est commune aux deux genres Amira et Khalilia, qui ne diffèrent, ainsi que nous l'avons fait remarquer dans notre précédent mémoire, que par le développement considérable du lobe dorsal de la bourse caudale du mâle chez le premier, tandis que ce lobe est très réduit chez le second. Dans ces deux genres les spicules du mâle sont grêles et très allongés, conformation en rapport avec la longueur du vagin chez la femelle. Parmi les Metastrongylidr, on retrouve dans le genre Metastrongylus Molin, 1861, une disposition analogue des spicules et du vagin.

Laboratoire de Parasilologie de la Faculté de médecine de Paris.

\section{ExPLICATION DE LA PLANCHE}

\section{Planche XXI}

Khalilia rhinocerotis N.-L. - 1. Femelle, grandeur naturelle. 2. Anatomie de la femelle. 3. Extrémité postérieure de la femelle, très grossie, montrant la longueur du vagin. 4. Ovéjecteurs vus à un fort grossissement. 5. EEuf. 